\title{
SYMMETRY OF TILINGS OF THE PLANE
}

\author{
CHARLES RADIN
}

\begin{abstract}
We discuss two new results on tilings of the plane. In the first, we give sufficient conditions for the tilings associated with an inflation rule to be uniquely ergodic under translations, the conditions holding for the pinwheel inflation rule. In the second result we prove there are matching rules for the pinwheel inflation rule, making the system the first known to have complete rotational symmetry.
\end{abstract}

We consider tilings of the Euclidean plane, $E^{2}$, by (orientation-preserving) congruent copies of a fixed finite set of prototiles. Prototiles are topological disks in the plane satisfying some mild restrictions on their shapes, as detailed below. Congruent copies of prototiles are called tiles, and a tiling is simply an unordered collection of tiles whose union is the plane and in which each pair of tiles has disjoint interiors.

We are concerned here with two constructions associated with a fixed finite set $S=\left\{P_{j}\right\}$ of prototiles, the most important of which is the set $X(S)$ of all tilings by tiles from $S$. In particular, we are interested in understanding the purest cases, in which all the tilings in $X(S)$ are "essentially the same"; we will define this precisely further on. Two examples are exhibited in Figures 1 and 2 on the next page, both with two prototiles; in Figure 1, $S=S_{K}$ produces only a checkerboard-like tiling (and all congruences), and in Figure $2, S=S_{P}$ produces the well-known tilings of Penrose $[3,4,6]$.

Tilings like those of Penrose are not usually invariant under any congruence of the plane (other than the identity), so to analyze their symmetries we introduce some elementary ergodic theory and another basic construction which can sometimes be associated with a prototile set $S$, the set $X^{F}(S)$ of tilings defined by an "inflation function" $F$. An "inflation rule" for $S$, if it exists, consists of a dilation $D_{F}$ of $E^{2}$ by some factor $\lambda_{F}<1$ and a finite set $\left\{C_{j k}\right\}$ of congruences of $E^{2}$, such that for each $P_{j} \in S$ we have

$$
P_{j}=\bigcup_{k} C_{j k} D_{F} P_{n_{k}}
$$

where the elements of each union have pairwise disjoint interiors. The inflation function $F$ associated with such a rule is defined on tiles (and then sets of tiles), with sets of tiles as values, as follows. If the tile $P$ is "of tile-type $j$ ", that is, $P=C P_{j}$ where $P_{j} \in S$ and $C$ is a congruence, then

$$
F: P \longrightarrow F(P) \equiv\left\{D_{F}^{-1} C C_{j k} D_{F} P_{n_{k}}\right\}
$$

(Intuitively, $P$ can be replaced by a set of "small-size tiles" by (1), which are

Received by the editors February 12, 1993.

1991 Mathematics Subject Classification. Primary 52C20, 58F11, 47A35.

Research supported in part by Texas ARP Grant 003658-113. 

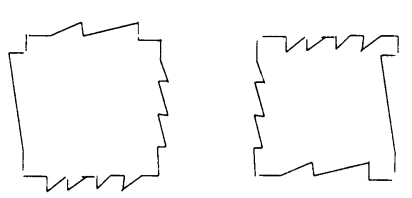

FIGURE 1
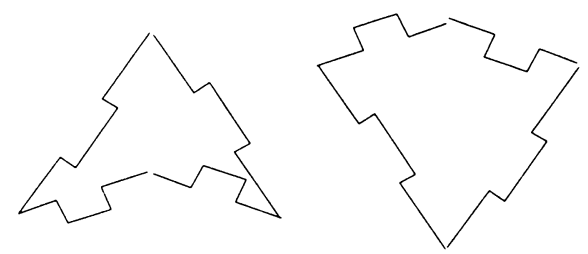

FIGURE 2

then expanded in (2) to original size by the inverse of the dilation. This process can obviously be applied to any collection of tiles-for example, a tiling-and can thus be iterated.)

The tilings $X^{F}(S)$ associated with the inflation function $F$ are then defined as those tilings $T$ such that each finite subcollection of $T$ is congruent to a subcollection of a set of tiles of the form $F^{r}(P)$ for some prototile $P$ and integer $r \geq 1$.

The construction $X^{F}(S)$ is mainly of interest when $F$ is a homeomorphism on it, since then $F$ defines a natural representation for the dilation $D_{F}$ as a map of $X^{F}(S)$ onto itself. (It is "natural" in that it extends the representation of the congruences to a larger subgroup of the conformal group.) One can then consider "symmetry" with respect to this hierarchical action, as we shall do. It is noteworthy that dilational (and rotational) symmetry is manifested not by the invariance of tilings themselves but rather of measures on tilings; that is, the action of the dilation (and rotations) is lifted to the set of (translation invariant Borel probability) measures on the tilings, and invariance is sought in this set. This is one reason for using the machinery of ergodic theory in the analysis of tilings.

We note the following examples of inflation rules.

(i) The square inflation rule $S$, given in Figure $3-S_{S}$ has one element;

(ii) The Robinson inflation rule $R$, given in Figure $4-S_{R}$ has two elements;

(iii) Conway's "pinwheel" inflation rule $C$, given in Figure $5-S_{C}$ has two elements.

(The inflation rule is one-to-one-and in fact a homeomorphism-on the associated space of tilings for the Robinson and pinwheel rules but not the square rule, for which it is four-to-one. Also, we really should distinguish between left- and right-handed triangles in Figure 4.) Each $X(S)$ (and $X^{F}(S)$ ) carries a natural metric structure (indicated below) and defines a dynamical system with $\mathbb{R}^{2}$ action, where $\mathbb{R}^{2}$ acts by simultaneous translation of the tiles in a tiling. Using this language, we note the unobvious fact [4] that the dynamical systems associated with $X^{R}\left(S_{R}\right)$ and $X\left(S_{P}\right)$ are topologically conjugate: there is a homeomorphism between $X^{R}\left(S_{R}\right)$ and $X\left(S_{P}\right)$ which intertwines the

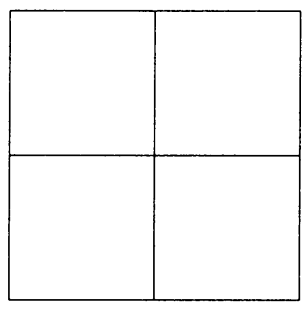

FIGURE 3 

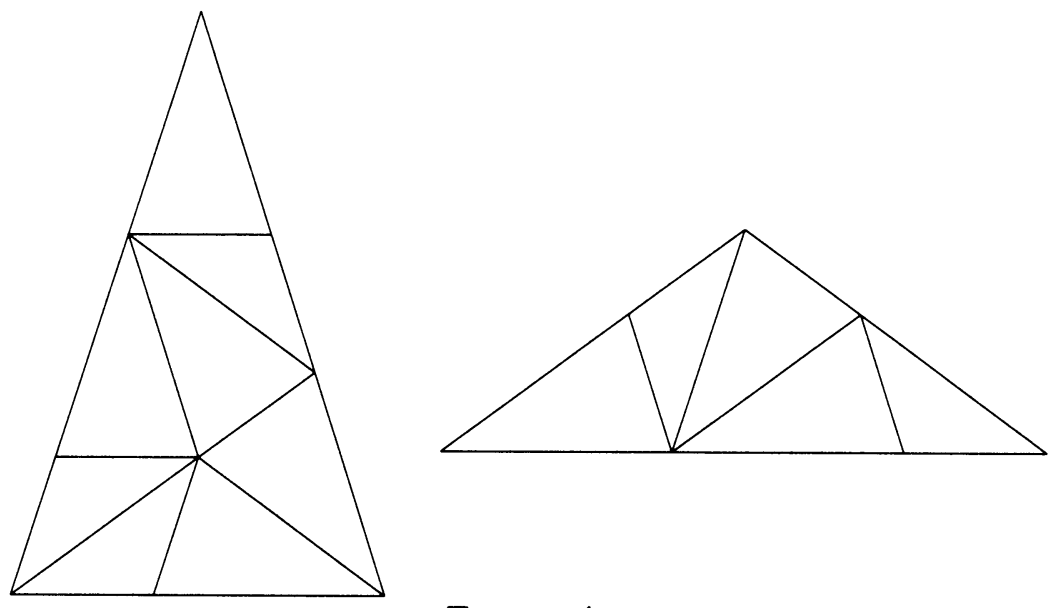

FIGURE 4

translations. It is immediate that those associated with $X^{S}\left(S_{S}\right)$ and $X\left(S_{K}\right)$ are topologically conjugate.

We now outline our results. Our first result gives conditions sufficient for the dynamical system associated with some $X^{F}(S)$ to be uniquely ergodic, the conditions being satisfied, for example, by $X^{C}\left(S_{C}\right)$. Unique ergodicity of a dynamical system, namely, the property that there is one and only one Borel probability measure on the space invariant under the group action-the space here being $X^{F}(S)$ and the group being $\mathbb{R}^{2}$-is useful because it means the dynamical system has a natural measure associated with it. (From Birkhoff's pointwise ergodic theorem [1], this is equivalent to having all the tilings being statistically identical [6].) The Penrose (and therefore Robinson) systems are not quite uniquely ergodic. However, each ergodic measure is invariant under rotation by $2 \pi / 10$ about any point of $E^{2}$, and the different measures are merely rotations of one another, by an angle in $(0,2 \pi / 10)$. So modulo this rotation there is a unique invariant measure for these systems. Furthermore, the ergodic measures for the Robinson system are each invariant under the dilation associated with Figure 4.

Our second result is the construction of a finite prototile set $\tilde{S}$ such that the dynamical system associated with $X(\widetilde{S})$ is uniquely ergodic, and metrically conjugate with that associated with $X^{C}\left(S_{C}\right)$. (Metric conjugacy-the existence of a measurable bijection modulo measure zero, which intertwines the dynamics-is somewhat weaker than topological conjugacy.) In other words, $X^{C}\left(S_{C}\right)$ plays a role for $X(\widetilde{S})$ similar to the one $X^{R}\left(S_{R}\right)$ plays for $X\left(S_{P}\right)$; in each case the former defines for the latter a hierarchical symmetry which,

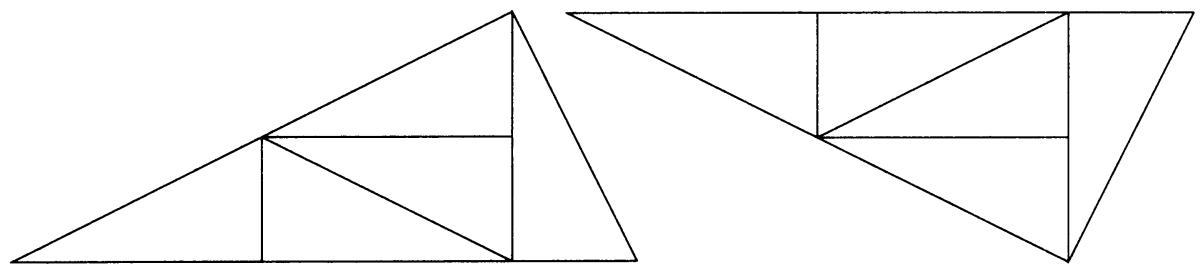

FIGURE 5 
for example, is manifested in a symmetry in the spectrum (discussed below) of the latter in each ergodic component. The pinwheel and Penrose systems differ significantly in their rotational symmetry: the Penrose system has the tenfold rotational symmetry of each of its ergodic components, while the pinwheel system, being uniquely ergodic, has full rotational symmetry. (The actions on a space of tilings of congruences of $E^{2}$, and of a dilation $D_{F}$-assuming $F$ is a homeomorphism on $X^{F}(S)$, of course-can be lifted to actions on the set of invariant measures of the dynamical system; so if a dynamical system is uniquely ergodic, its measure must be invariant under such actions.) The symmetries of the spectrum of tiling systems - in particular, the Penrose tilings-have been of major importance in their connection with theories of the structure matter, for example, quasicrystals [6].

We now state our assumptions and results more fully. We assume that the prototiles in $S$ satisfy the following conditions, besides being homeomorphs of the closed unit disk:

- $X(S)$ is nonempty;

- in the tilings being considered, the boundary of each tile can be covered by tiles in only finitely many ways, up to congruence;

- each prototile $P$ has small surface-to-volume ratio in the sense that:

$$
\frac{\operatorname{area}\{x \in t P:\|x-y\| \leq 1 \text { for some } y \in \partial(t P)\}}{\operatorname{area}\{t P\}} \longrightarrow 0
$$

as the expansion factor $t \rightarrow \infty$.

A metrizable topology is put on the space $X(S)$ or $X^{F}(S)$ of tilings with the following neighborhood basis $\left\{N_{T}(\epsilon): \epsilon>0\right\}$ of each tiling $T: N_{T}(\epsilon)$ consists of all tilings $T^{\prime}$ such that within the circle in the plane centered at the origin and of radius $1 / \epsilon$, wherever $T$ has a tile, so does $T^{\prime}$, within distance $\epsilon$ in the Hausdorff metric on compact sets. It is known that $X(S)$ and $X^{F}(S)$ are compact in this topology and that the actions of translations $\mathbb{R}^{2}$ on $X(S)$ and $X^{F}(S)$ are continuous [9]. Given the above then, our results are the following.

Theorem 1 [8]. Assume given some $X^{F}(S)$ as above, and assume there is some $r \geq 1$ such that:

(a) for each prototile $P, F^{r}(P)$ contains tiles of every tile-type;

(b) for some prototile $P, F^{r}(P)$ contains two tiles $P^{\prime}, P^{\prime \prime}$ of the same tile-type, whose relative rotation is irrational with respect to $\pi$.

Then $\left(X^{F}(S), \mathbb{R}^{2}\right)$ is uniquely ergodic.

Theorem 2 [7]. There is a finite set $\tilde{S}$ of prototiles such that $\left(X(\tilde{S}), \mathbb{R}^{2}\right)$ is uniquely ergodic and metrically conjugate to $\left(X^{C}\left(S_{C}\right), \mathbb{R}^{2}\right)$.

We note that $X^{C}\left(S_{C}\right)$ satisfies the hypotheses of Theorem 1 (with $r=2$ ) and close with a few comments on these results of a more technical nature.

Much of the symbolic (substitution) dynamics of hierarchical structures makes use of a square matrix $A$, of which $A_{j k}$ is the number of times tiletype $j$ is associated, by the inflation function $F$, with tile-type $k$. To prove Theorem 1 , this matrix is generalized to a family $A[\mathrm{~m}]$ of matrices for which $A[m]_{j k}$ is the sum $\sum_{n} e^{i m a_{n}(j, k)}$, over all tiles of type $j$ contained in $F\left(P_{k}\right)$, 
where $a_{n}(j, k)$ is the angle of rotation of the tile compared to the defining prototile of its type.

This generalization allows us to keep track of rotational information, and we prove unique ergodicity by using Weyl's criterion on uniform distribution as it is used in elementary treatments of rotations of the circle [1]. The natural way in which the important matrix $A$ generalizes is evidence to us that much of symbolic substitution dynamics can be generalized to tilings $X^{F}(S)$.

The import of Theorem 2 is also, in part, its relation to symbolic dynamics. The systems $X(S)$ are natural generalizations of symbolic systems of finite type with $\mathbb{Z}^{2}$ action; for example, they play roughly the same role for statistical mechanics in Euclidean spaces as systems of finite type do for lattice gas statistical mechanics [6]. Of particular importance, for example, in theories of material structure are the spectra of such dynamical systems. To see why the symmetries of the dynamical system are relevant for this, note that if a rotation or dilation $W$ preserves the ergodic probability measure $m$ of the dynamical system, then, as shown in [8], the spectral projection $E_{\Delta}$ of the translations, associated to a set $\Delta \subset \mathbb{R}^{2}$, is unitarily equivalent, in the Hilbert space defined by $m$, to $E_{W(\Delta)}$. (See [2] for explicit connections between dynamics spectra and X-ray spectra of scatterers.)

Another aspect of Theorem 2 is its relation to the work of S. Mozes, who proved in [5] that a rather general class of symbolic substitution dynamical systems with $\mathbb{Z}^{2}$ action are of finite type; our example is, we hope, the first step in a parallel theorem for tilings of the plane.

\section{REFERENCES}

1. I. Cornfeld, S. Fomin, and Ya. Sinai, Ergodic theory, Springer-Verlag, New York, 1982.

2. S. Dworkin, Spectral theory and X-ray diffraction, J. Math. Phys. (to appear).

3. M. Gardner, Extraordinary nonperiodic tiling that enriches the theory of tiles, Sci. Am. (USA) 236 (1977), 110-119.

4. B. Grünbaum and G. C. Shephard, Tilings and patterns, Freeman, New York, 1986.

5. S. Mozes, Tilings, substitution systems and dynamical systems generated by them, J. Analyse Math. 53 (1989), 139-186.

6. C. Radin, Global order from local sources, Bull. Amer. Math. Soc. (N.S.) 25 (1991), 335-364.

7. _ The pinwheel tilings of the plane, Ann. of Math. (2) (to appear).

8. __ Space tilings and substitutions, Univ. of Texas, preprint.

9. C. Radin and M. Wolff, Space tilings and local isomorphism, Geom. Dedicata 42 (1992), $355-360$.

Mathematics Department, University of TeXas, Austin, TeXas 78712

E-mail address: radin@math.utexas.edu 